\title{
USRP Based Digital Audio Broadcasting Using OFDM in Virtual and Remote Laboratory
}

\author{
https://doi.org/10.3991/ijoe.v15i13.8761 \\ Nagarjuna Telagam ${ }^{(\bowtie)}$, \\ GITAM University, Bangalore, India \\ nagarjuna 473@gmail.com \\ Padma Charan Sahu \\ GIET University, Gunupur, India \\ Sunita Panda \\ GITAM University, Bangalore, India \\ Nehru Kandasamy \\ Institute of Aeronautical Engineering, Hyderabad, India
}

\begin{abstract}
Digital Audio Broadcasting (DAB) is an amazing technology, achieving its promise of certainly delivering high quality digital audio in the most vindicate mobile and fixed receiver environments. The radio signal processing capability can be provided by Universal software radio peripheral (USRP) hardware. The received signal frequency can be controlled and transmitting the signal through wireless is possible by installing NI USRP utility configuration. The programmable USRP was running the VI snippet program in block diagram panel with radio companion integrated through USB cable on windows 7 64. With the parameters such as IQ rate, transmitter gain, carrier frequency along with device name which is given as ni2901_1 the students can able to tune the radio signal. DAB technology is integrated with USRP device using prominent orthogonal frequency division multiplexing (OFDM) technique for promise delivery of high quality audio signal. DAB works under four transmission modes in this paper. In this paper RF0 module is used USRP device is connected with dual band vertical antenna with 2.4 and $5 \mathrm{GHz}$ frequency as Tx1. This transmitting VI snippet program will support all file formats such as.wav, $\mathrm{mp} 3$, etc. the intention of the paper is to transmit the real time data through long distance using DAB technology in remote laboratory. The proposed system is implemented in virtual lab so that it can be accessed by any user from anywhere. This system is successfully tested on institute of aeronautical engineering virtual lab for the purpose of exposing the Radio frequency tools and signal processing operations for students.
\end{abstract}

Keywords-USRP, LabVIEW, DAB, VI, AUDIO. 


\section{Introduction}

Virtual and remote laboratories reflect a movement among education institutions to make the equipment and elements of a physical science laboratory more easily available to learners from any location, via the web. Users are able to manipulate the equipment and watch the activities unfold via a webcam on a computer or mobile device. This provides students with a realistic view of system behavior and allows them access to professional laboratory tools from anywhere, whenever they need. Additionally, remote labs alleviate some financial burden for institutions as they can forgo purchasing specific equipment and use the remote tools that are at their disposal.[10] Laboratory experimentation plays an essential role in engineering and scientific education. Virtual and remote labs reduce the costs associated with conventional hands-on labs due to their required equipment, space, and maintenance staff. Furthermore, they provide additional benefits such as supporting distance learning, improving lab accessibility to handicapped people, and increasing safety for dangerous experimentation. [2]. It is envisioned that all broadcasting systems will be digitalized in future. New services and more information require high data rates for broadcasting systems. There are many digital audio broadcasting standards in the world, such as DAB (Digital Audio Broadcasting) in Band III and Band L [3].Software radios are emerging as platforms for multiband multimode personal communications systems. Radio etiquette is the set of RF bands, air interfaces, protocols, and spatial and temporal patterns that moderate the use of the radio spectrum [4]. Software-defined radios (SDRs) promise unprecedented flexibility, but their architecture has proven to be a challenge for MAC protocols [5]. After being the subject of speculation for many years, a software-defined radio receiver concept has emerged that is suitable for mobile handsets. A key step forward is the realization that in mobile handsets, it is enough to receive one channel with any bandwidth, situated in any band. Thus, the front-end can be tuned electronically [6].A system and method for providing one hardware platform to implement multiple wireless communication standards, services and applications. The kernel oriented macro based Software defined radio architecture provides a configurable and programmable hardware platform to implement multiple wireless standards, services and applications [7]. The experiments based on USRP device was implemented in virtual and remote lab was explained [8] and same experiment was done for the purpose of students with FM transceiver with large file capabilities [9]. Digital audio signal is broadcasted in rural area with gfdm transceiver using software defined radio and plotted the graphs [11]. 


\section{Implementation}

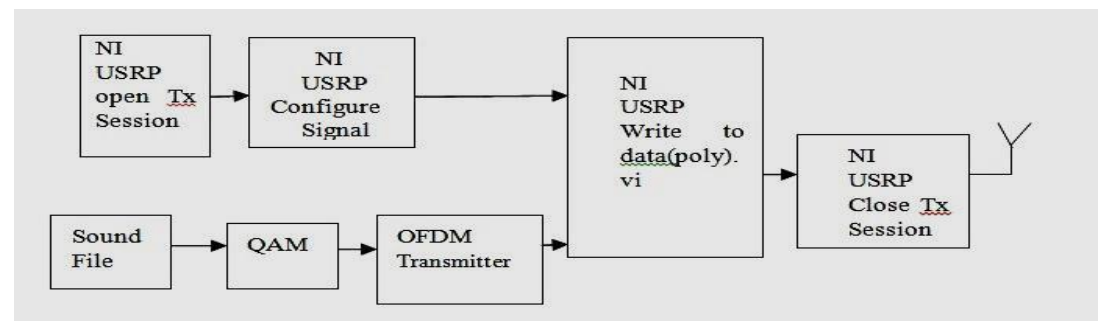

Fig. 1. Proposed System of DAB using NI USRP 2901

Figure 1 shows the implementation steps of Digital audio broadcasting system using USRP 2901. The session was opened using vi program which is inbuilt. Then we configure the signal with active antenna, gain in $\mathrm{dB}$, carrier frequency of the antenna using NI USRP configure signal utility. We have used input as sound file and converted the analog data to digital signal using the icons in the lab view. The QAM uses only ones and zeros as input and then output of signal mapper block is connected to OFDM Transmitter which has nearly 10 blocks in it. OFDM is an amazing technology with many applications and supports orthogonality to avoid the interferences between the signals. QAM mapper converts bits to symbols. The two inputs are given to NI USRP write to data vi with different data types of data. Usually pink color data is coming from configure signal VI and orange color data which is of signal is connected to write to data VI. Finally the verto antenna which supports tri bands is used to transmit the data.

\subsection{Experimental set-up with Tri band antenna}

Figure 2 shows the experimental set up. The set up usually connects the USRP 2901 using USB Cable. After connecting this device the PC shows the NI configuration device window in which the name of USRP device will appear. The same name should use at the front panel of the program to run the VI. The figure shows red color blinking in the device which means the data is transmitting if the device shows green color the data is receiving.

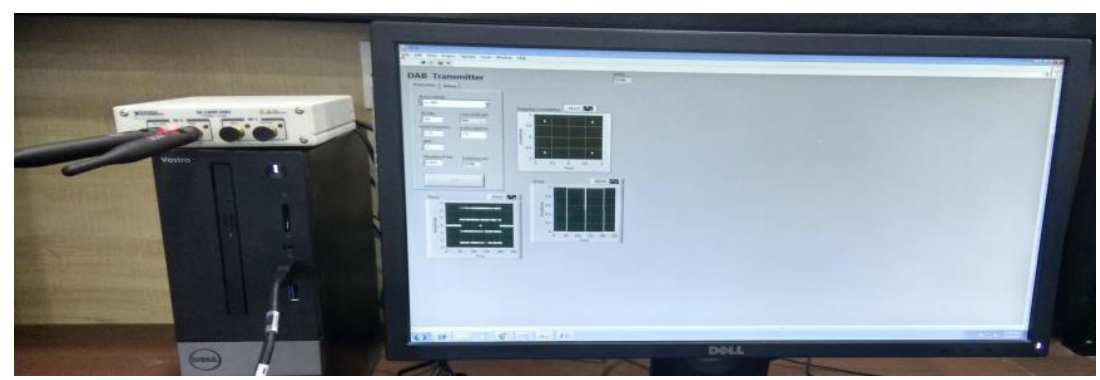

Fig. 2. Experimental setup of USRP Transmitting Audio Signal 
Connect ni USRP device to computer using USB cable and AC/DC power supply cable. Configuration of ni USRP devices follows:

- Select Start» All Programs» National Instruments» NI-USRP»NI-USRP Configuration Utility to open the NI-USRP Configuration Utility.

- Your device should appear in the list on the left side of the tab, similar to the following figure.

- If you have multiple devices, verify that you selected the correct device. The device ID of the selected device displays in the Selected Device ID textbox.

\subsection{Working operation}

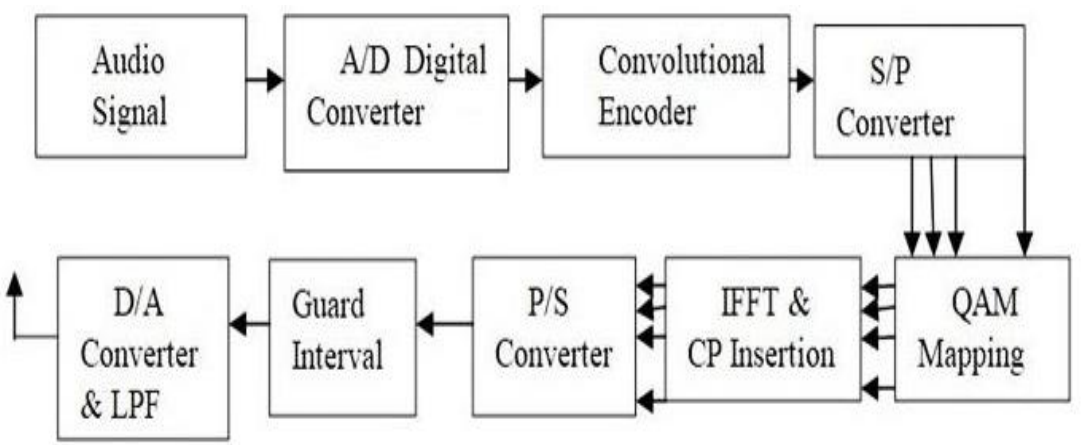

Fig. 3. VI Programs used in LabVIEW

Figure 3 is an glimpse of the icons used in transmitter program. Some of the VI are already inbuilt in the labview. Here the input is audio signal with different formats. We have converted the audio file into digital format ie one's and zero's because the QAM signal mapper accepts only digital data to modulate. According to DAB block diagram the converted digital bits need to encoded. Here we have used convolutional encoder icon which is already availble in RF Communications labview. Then we have converted the serial data to parallel format using subset array. Then we have mapped the bits to symbols and applied IFFT VI to the parallel data. This icon converts frequency domain data into time domain data with less number of multiplications. We have used inbuilt VI named cyclic insertion to avoid Intersymbolic Interference between the symbols. Then we have used array icons to convert this time domain parallel data to serial data. Finally we have used guard interval block to avoid the Intercarrier Interference noise. Here table 1 is shown below which is used for simulation. The simulation parameters are used in labview program. The carrier frequency used is $1.5 \mathrm{GHz}$. 
Table 1. Parameters used in VI program i.e.DAB Transmission Modes

\begin{tabular}{|c|c|c|c|c|}
\hline No of Subcarriers & Subcarrier spacing & Symbol time & Carrier Frequency & $\begin{array}{c}\text { Transmitter separa- } \\
\text { tion }\end{array}$ \\
\hline 192 & $8 \mathrm{kHz}$ & $155.8 \mu \mathrm{S}$ & $<3 \mathrm{GHz}$ & $<12 \mathrm{Km}$ \\
\hline 1536 & $1 \mathrm{KHz}$ & $266.2 \mu \mathrm{S}$ & $375 \mathrm{MHz}$ & $<5 \mathrm{Km}$ \\
\hline 384 & $4 \mathrm{KHz}$ & $666.6 \mu \mathrm{S}$ & $1.5 \mathrm{GHz}$ & $<10 \mathrm{Km}$ \\
\hline 768 & $2 \mathrm{KHz}$ & $133.3 \mu \mathrm{S}$ & $750 \mathrm{MHz}$ & $<20 \mathrm{Km}$ \\
\hline
\end{tabular}

\section{VI Program Using LabVIEW}

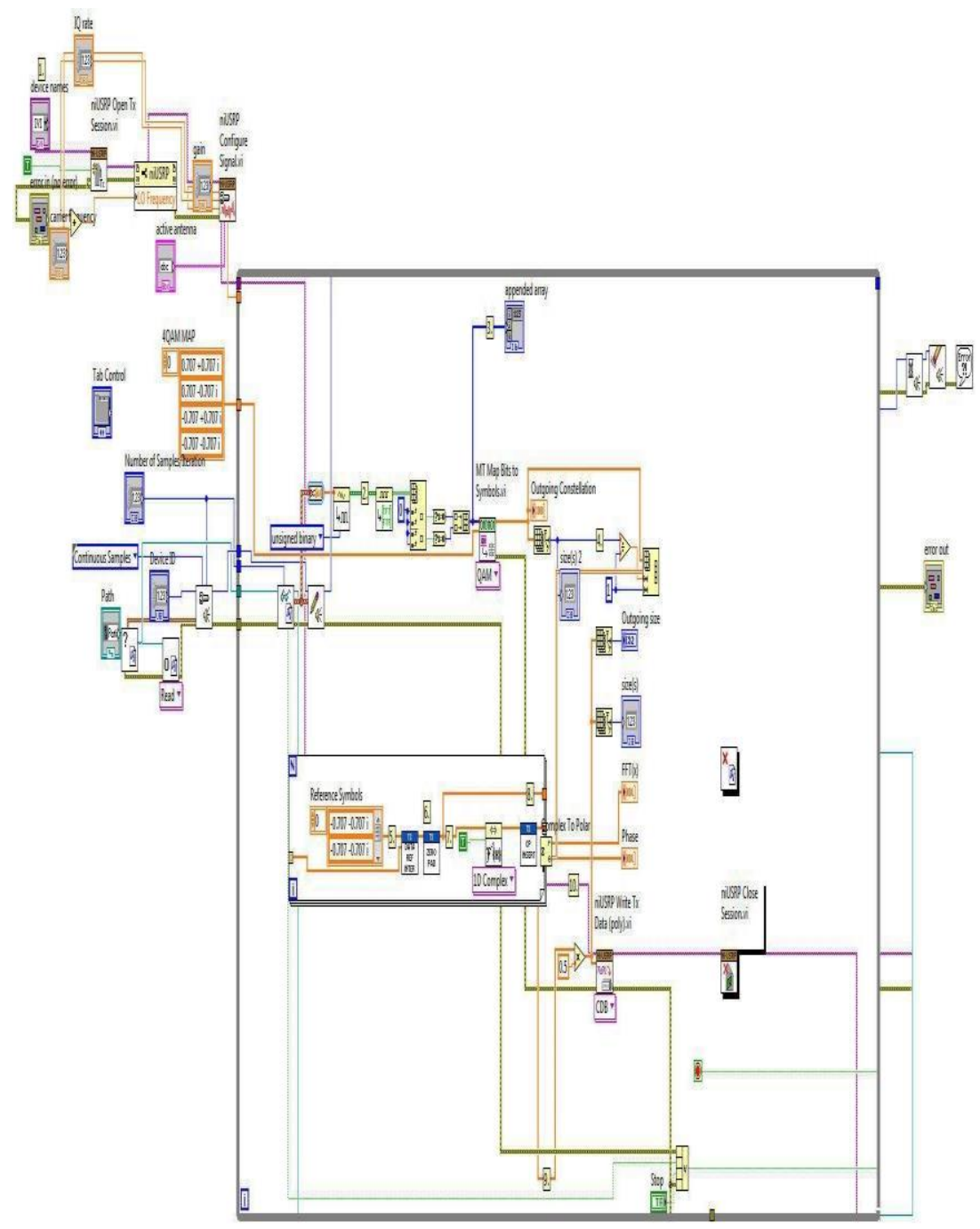

Fig. 4. Digital Audio Broadcasting VI Program used in Block Diagram 
The figure 4 shows the sound file is taken as input with the help of path. The icons such as sound read, sound write is used. The device ID is used and we have configured the signal. We have converted the analog signal to digital signal using dynamic data type icon along with analog to digital. VI, and given to array subset. We have checked the data using appended array and confirmed the output which has only ones and zeros. 4-QAM mapping is used and we have converted the serial data to parallel data with the help of arrays. This parallel data is given to IFFT.VI, which converts frequency domain representation to time domain representation. We have plotted the response in both phase plot and magnitude plot with the help of graph palate the students can able to analyze the different parallel data as shown in figure 7 . We have connected the output of IFFT along with guard band interval to Ni usrp write to poly.VI which transmits the audio signal through Verito tri band antenna.

\section{$4 \quad$ Results and Discussions}

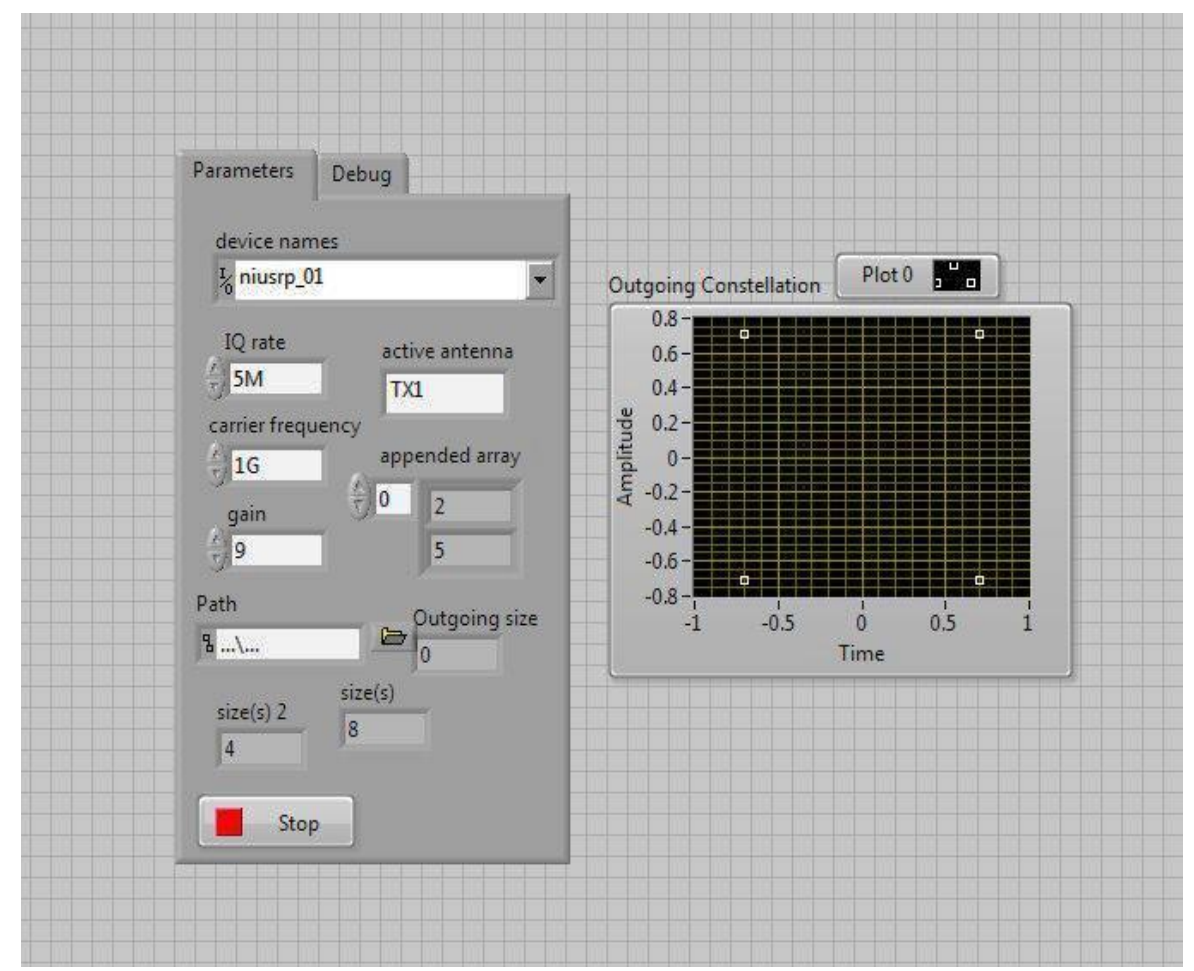

Fig. 5. Screen shot of USRP Configuration in front panel 


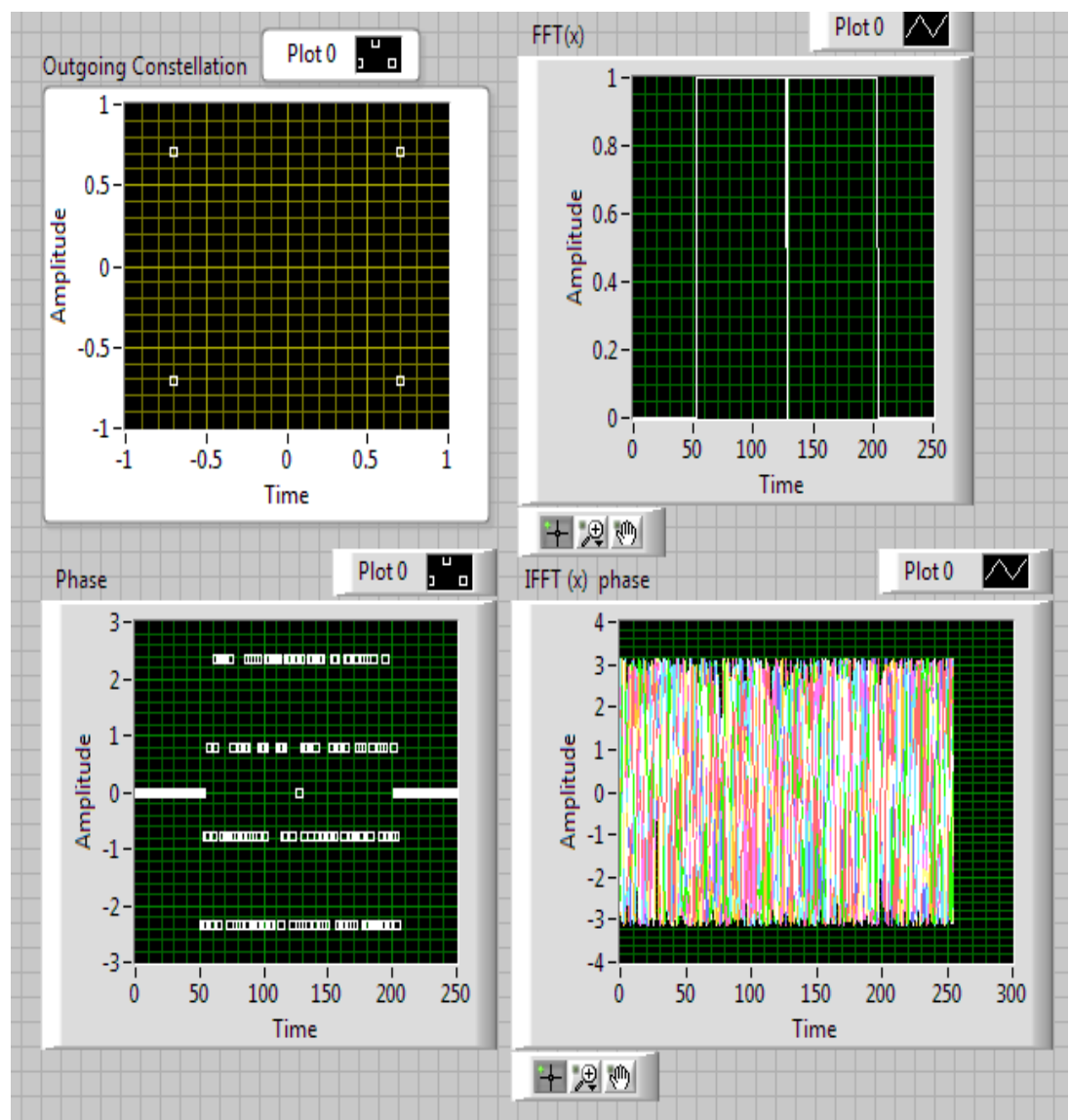

Fig. 6. Screen shot of Results in Front panel.

Figure $5 \& 6$ shows the screen shots of front panel. In Figure 8 the one on the top left is QAM mapping with 4 reference symbols. The I/Q mapping block takes each symbol $s_{k}$ and maps it to a corresponding complex baseband value $C_{k}$ in the complex plane. These values are usually chosen to be as different (or far apart) as possible so that at the receiver we can discern as clearly as possible what was transmitted. A nice way to represent the mapping of symbols to complex baseband I/Q values is with a constellation diagram. The second on the top right corner is FFT(x) signal and third one to the left below is the phase response of the signal. The last one is IFFT(x) which gives the digital audio signal in time domain.

\section{Conclusion}

Thus the Digital Audio Broadcasting system using OFDM is implemented using a USRP 2901 with tri-band antenna. The real time data is send over $1 \mathrm{GHz}$ frequency with distance of nearly $1 \mathrm{Km}$. The demand for high data rate wireless communication 
has been increasing dramatically over the last decade. OFDM, which is the most emerging multi-carrier modulation technology of this era, can solve this problem significantly. This paper has provided an analysis of OFDM behaviors, principles and analyzed different techniques error reduction and frequency offset estimation that improve performance of OFDM for wireless communications Lastly the simulation results found with the help of working setup of OFDM system implementation using LabVIEW software and USRP hardware which conclude the successful transmission and reception of information bit using 4 QAM techniques with equalized data and amplitude after OFDM receiver.

\section{References}

[1] Heradio, Ruben, Luis de la Torre, Daniel Galan, Francisco Javier Cabrerizo, Enrique Herrera-Viedma, and Sebastian Dormido. (2016). Virtual and remote labs in education: A bibliometric analysis. Computers \& Education, 98: 14-38. https://doi.org/10.10 16/j.compedu.2016.03.010

[2] Di, Na, PengGao, Ge Wan, Jilong Li, and Jinwen Li. (2010). A common SDR platform for digital audio broadcasting system. In Image and Signal Processing (CISP), 3rd International Congress on, 8: 3708-3711. https://doi.org/10.1109/cisp.2010.5647099

[3] Mitola, Joseph, and Gerald Q. Maguire. (1999). Cognitive radio: making software radios more personal. IEEE personal communications 6, 4: 13-18. https://doi.org/10.1109/98.788210

[4] Nychis, George, ThibaudHottelier, Zhuocheng Yang, SrinivasanSeshan, and Peter Steenkiste.(2009). Enabling MAC Protocol Implementations on Software-Defined Radios. In NSDI, 9:91-105.

[5] Abidi, Asad A. (2007). The path to the software-defined radio receiver. IEEE Journal of Solid-State Circuits, 42, 5: 954-966. https://doi.org/10.1109/jssc.2007.894307

[6] Ting, Pangan, Hui-Ming Wang, and Nan-Sheng Huang.(2006). Software defined radio (SDR) architecture for wireless digital communication systems. U.S. Patent 7,151,925, issued.

[7] Kandasamy, N., Telagam, N., VR, S. R., \& Arulananth, T. S. (2017). Simulation of Analog Modulation and Demodulation Techniques in Virtual Instrumentation and Remote Lab. International Journal of Online Engineering (iJOE), 13(10): 140-147. https://doi.org/10.3991/ijoe.v13i10.7575

[8] Nagarjuna, T., S. Lakshmi, and K. Nehru.(2019), USRP 2901-based SISO-GFDM transceiver design experiment in virtual and remote laboratory. The International Journal of Electrical Engineering \& Education (2019): 0020720919857620. https://doi.org/10.1177/0020720919857620

[9] Somanaidu, U., Nagarjuna Telagam, K. Nehru, and N. Menakadevi.(2018) USRP 2901 Based FM Transceiver with Large File Capabilities in Virtual and Remote Laboratory. International Journal of Online Engineering (iJOE), 14, (10): 193-200. https://doi.org/10.3 991/ijoe.v14i10.8283

[10] https://www.nmc.org/horizon_topic/virtual-and-remote-laboratories/

[11] Nagarjuna Telagam, S.Lakshmi, K.Nehru, (2019). Digital audio broadcasting based gfdm transceiver using software defined radio, International journal of Innovative technology and Exploring Engineering, 8, (5): 273-281. 


\section{$7 \quad$ Acknowledgement}

The corresponding author would like to thank institute of aeronautical engineering, Hyderabad for establishing virtual instrumentation lab in the college. He would like to thank his personage, dignitary nd for the best wishes.

\section{Authors}

Nagarjuna Telagam is currently working in ECE Department, GITAM University, Bangalore, India. His topics of interest are OFDM, GFDM and wireless sensor networks. He published 25 papers in Scopus indexed Journals and 4 SCIE journals. He got CLAD certification from National Instruments. Currently he is Ph.D. Research Scholar in Sathyabama University Chennai. He was awarded Anna University rank holder (20) for M.E degree in 2014. He has nearly 6 years of experience in teaching and research.

Padma Charan Sahu is currently working as assistant professor in kalam institute of technology since 2010. He is currently pursuing Ph.D. in GIET University, Gunupur. He can be reached at kit.padma@gmail.com

Sunita Panda is with Electronics and Telecommunication Engineering department, GITAM University, Bangalore campus. Currently she is Assistant Professor, Her areas of interest are soft computing, channel equalization, Digital Signal processing. She can be reached at sunita.nano@gmail.com

Nehru Kandasamy is with Electronics and Communication Engineering, Institute of Aeronatuical Engineering, Hyderabad, India. He can be reached at nneh$\underline{\text { ruk@gmail.com }}$

Article submitted 2018-04-19. Resubmitted 2019-07-10. Final acceptance 2019-07-11. Final version published as submitted by the authors. 